\title{
NARRATIVAS Y ENFOQUE BIOGRÁFICO. USOS, ALCANCES Y DESAFÍOS PARA LA INVESTIGACIÓN INTERDISCIPLINARIA
}

\author{
NARRATIVES AND BIOGRAPHICAL APPROACH. USES, SCOPE AND CHALLENGES \\ FOR INTERDISCIPLINARY RESEARCH
}

NARRATIVA BIOGRÁFICA E ABORDAGEM. USOS, ÂMBITO E DESAFIOS

PARA A PESQUISA INTERDISCIPLINAR

\author{
Gabriela Rubilar Donoso \\ Universidad de Chile. Santiago de Chile, Chile. \\ grubilar@uchile.cl \\ ORCID: 0000-0002-4635-9380
}

DOI: https://doi.org/10.22235/ech.v6iEspecial.1453 Recibido: $15 / 06 / 2017$ Aceptado: 09/09/2017

\section{RESUMEN}

Este artículo revisa los alcances y potencialidades de la investigación con enfoque biográfico y el papel que adoptan, dentro de este enfoque, las narrativas que dan cuenta de los procesos vividos por los sujetos. Pone especial énfasis en el uso de las narrativas para la construcción de relatos, historias de vida y testimonios, aportando elementos para una discusión acerca de su uso y revalorización como enfoque de investigación e intervención. El artículo se escribe desde una perspectiva interdisciplinaria, ya que se reconocen los aportes de este enfoque a distintas disciplinas de las ciencias sociales, humanas y ciencias de la salud. Se analizan las corrientes que han influido en los estudios de enfoque biográfico, considerando aspectos históricos y epistemológicos. Lo anterior es especialmente relevante en el caso de aquellas disciplinas que están relacionadas con los cuidados, como la Enfermería o el Trabajo Social, que trabajan con relatos de sujetos que enfrentan situaciones de dolor o enfermedad. El enfoque biográfico narrativo permite rescatar sus historias y contribuir a la memoria de aquellas personas dispuestas a narrar sus experiencias. El artículo finaliza mostrando los usos contemporáneos de este enfoque tanto en el campo de la investigación como en la intervención social. Discute acerca de los desafíos actuales de este enfoque y en sus posibilidades de combinación con dispositivos multimedia y el uso de tecnologías de información.

Palabras claves: Biografía, Narrativas Personales, Investigación Cualitativa, Investigación Interdisciplinaria, Profesionalismo.

\section{ABSTRACT}

This article reviews the scope and potential of research done using a biographical approach and the role that this approach adopts in giving voice to experiences lived by the subjects. Special emphasis is placed on the use of narratives to construct life stories, histories and testimonies, incorporating elements for a discussion about their use and enhancement as an approach for research and intervention. This article is written from an interdisciplinary perspective, recognizing the strengths of this approach that can be applied to diverse disciplines within social sciences, humanities and health sciences. This paper analyzes the trends that have influenced in studies from a biographical approach, considering historical and epistemological aspects. This is particularly relevant for disciplines related to human care, such as Nursing or Social Work that deal with narratives of participants who have faced situations of pain or illness. The narrative-biographical approach allows us to retrieve these histories and to contribute to the memories of people willing to narrate their experiences. The article concludes by examining the contemporary uses of this approach both in research and in social interventions. Current challenges related to this approach are discussed and also the possibility of combining it with multimedia devices and the use of information technology.

Keywords: Biography, Personal Narratives, Qualitative Research, Interdisciplinary Research, Professionalism.

\section{RESUMO}

Este artigo discorre sobre o potencial da pesquisa a partir de uma abordagem biográfica e do rol que adoptam dentro deste enfoque as narrativas que dão conta dos processos vivenciados pelos sujeitos. Enfatiza, especialmente, no uso das narrativas na construção de relatos, histórias de vida e testemunhas, fornecendo elementos para uma discussão sobre seu uso e sua apreciação como enfoque de pesquisa e intervenção. $\mathrm{O}$ artigo está escrito a partir de uma perspectiva Interdisciplinar, visto que reconhece os aportes desse enfoque, aplicado a diferen- 
tes disciplinas das ciências sociais, humanidades e ciências da saúde. São revisadas as correntes que influenciaram os estudos de tipo biográfico, no que tange a quesitos históricos e epistemológicos. Isso é especialmente relevante no caso de aquelas disciplinas, relacionadas com os cuidados, entre os quais: Enfermagem ou Trabalho social, que trabalham com histórias de indivíduos que enfrentam situações de dor ou doença. A abordagem biográfica narrativa permite resgatar suas histórias e contribuir para a memória de aqueles dispostos a relatar suas experiências. $\mathrm{O}$ artigo termina mostrando os usos contemporâneos dessa abordagem, tanto na pesquisa quanto na intervenção social. Discute os desafios atuais desta abordagem e suas possibilidades de combinação com dispositivos de multimídia e uso de tecnologias da informação.

Palavras-chave: Biografia, Narrativas Pessoais, Pesquisa Qualitativa, Pesquisa Interdisciplinar, Profissionalismo.

\section{INTRODUCCIÓN}

Este artículo revisa los alcances y potencialidades de la investigación con enfoque biográfico, considerando como problema de investigación los usos y posibilidades que esta perspectiva ofrece a la investigación interdisciplinar. Se pone especial énfasis en la relación entre el enfoque biográfico y el uso que al interior de esta perspectiva alcanzan las narrativas y los relatos construidos desde o con los propios sujetos investigados.

Algunos de los contenidos de este artículo fueron presentados en la Escuela de Verano en metodologías cualitativas para la transformación social en zona fronteriza, realizada en el mes de enero de 2017 en la Universidad de Tarapacá. Esta Escuela tenía como propósito actualizar y compartir conocimientos sobre las metodologías cualitativas en sus distintos momentos y desde distintas aproximaciones, y es en ese contexto que se exponen los resultados de una investigación que reflexiona acerca del uso de las narrativas para la construcción de relatos, historias de vida y testimonios, aportando elementos para una discusión acerca de su uso y revalorización como enfoque de investigación e intervención (1).

Se parte del supuesto que el enfoque biográfico tiene una larga tradición en las ciencias sociales; no obstante, su uso y despliegue como perspectiva de investigación no siempre es reconocido en todos los campos disciplinarios. Se piensa también que su uso renovado y dinamizado a través de tecnologías de información y comunicación puede aportar nuevas perspectivas de análisis, al mismo tiempo que nutre a otras disciplinas de las ciencias sociales y humanas.

Particularmente en este artículo se pone especial atención a las posibilidades de desarrollo de este enfoque desde disciplinas que están relacionadas a los cuidados, como el Trabajo Social y la Enfermería, rescatando las historias y narrativas de los sujetos con los cuales estos profesionales trabajan, los que muchas veces enfrentan situaciones de dolor o sufrimiento que merecen ser narradas y analizadas desde esta perspectiva.

\section{METODOLOGÍA}

En esta investigación se realiza una revisión bibliográfica y documental de las principales corrientes que han influido en los estudios del enfoque biográfico, considerando aspectos históricos y epistemológicos.

Habitualmente se interroga si la perspectiva biográfica es un enfoque, un método o una técnica. En este artículo, la perspectiva biográfica es considerada un enfoque, que como tal, forma parte de los territorios de la investigación cualitativa. Como enfoque la perspectiva biográfica considera aspectos teóricos, epistemológicos y metodológicos específicos de su desarrollo.

Uno de estos elementos distintivos de esta perspectiva es que el enfoque biográfico se construye a partir de los relatos personales que los sujetos narran a los investigadores. Estas narraciones pueden dar cuenta de toda la vida de una persona o ser una narración más acotada a ciertos sucesos o acontecimientos de interés para quien investiga.

Se considera como punto de partida de los estudios biográficos el trabajo clásico de William Thomas y Florian Znaniecki (2). Este estudio, cuya publicación está próxima a cumplir cien años, nos permite afirmar que los estudios biográficos tienen una larga tradición en las ciencias sociales, que se remonta a los inicios de la Escuela de Sociología de Chicago $(3,4,5)$. La vigencia de esta obra nos recuerda el influjo que alcanza en la actualidad lo biográfico como enfoque de investigación y las distintas derivadas que esta perspectiva ha tenido no sólo en la propia Escuela de Chicago, sino en visiones más contemporáneas como las desarrolladas por la Escuela polaca de la Universidad de Łódź.

En el período fundacional de los estudios desarrollados bajo esta perspectiva, distintos investigadores del departamento de sociología de la Escuela de Chicago, dirigidos por Park, examinaron la realidad social de su tiempo, con un esquema de análisis que interpelaba a elementos estructurales, conectando los problemas sociales con las experiencia vitales, y esto, a su vez, con la estructura social, articulando teoría y datos (6).

Las reflexiones desarrolladas posteriormente en torno a una genealogía de la identidad profesional de las pioneras del Trabajo Social nos permiten comprender mejor las influencias y relaciones que, en ese período, se constituyeron entre los exponentes de la Escuela de Chicago y el colectivo de mujeres que profesionalizó el Trabajo Social en Estados Unidos a finales del siglo XIX e inicios del XX $(7,8)$.

La Hull House se convirtió en un espacio privilegiado para el estudio y la intervención, haciendo de la perspectiva biográfica una de sus principales herramientas de trabajo. Observamos cómo tempranamente las mujeres de la Hull House usan activamente este enfoque combinado con otras técnicas como el mapeo o las cartografías, que hasta el día de hoy constituyen un valioso aporte para el estudio de las comunidades de migrantes y sus condiciones sociales (9). 
Los vínculos entre Hull House y el trabajo de Thomas y Znaniecki son fuertes, ya que ambos comparten como benefactor y mecenas a Charles Hull y a su prima Helen Culver, con quien Thomas mantiene una nutrida correspondencia que da cuenta de la trastienda de su investigación. Aspecto que va a mostrar la forma cómo esta perspectiva se instala tempranamente en las nacientes disciplinas de las ciencias sociales (10).

Después de la Segunda Guerra Mundial los estudios biográficos decayeron notoriamente, tanto en sociología como en antropología. Para Bertaux la Segunda Guerra Mundial desplazó el centro investigativo hacia el funcionalismo parsoniano y el análisis de encuestas, lo que redujo las demás formas de observación y de teorización (11).

Esta suerte de "abandono" de los estudios biográficos queda registrado en los trabajos de Norman Denzin y Juan Francisco Marsal, quienes varias décadas más tarde retoman estos estudios en sincronía con el auge que experimentan los enfoques cualitativos desde fines de los años 70 en adelante $(12,13)$.

Es en este contexto que se reconoce en este artículo a Daniel Bertaux como uno de los mayores referentes del método biográfico, y quizás quien mejor representa en este período un impulso renovador que terminó borrando los límites entre la sociología, la antropología y la historia (14).

Este autor sostiene que el enfoque basado en las historias de vida y los relatos biográficos tienen un potencial analítico que va mucho más allá de su ya conocida capacidad exploratoria, afirmación que posteriormente recoge en su propuesta de análisis comprensivo, la que permite no sólo describir el funcionamiento microsocial, sino también extraer conclusiones que sobrepasan lo particular.

Su visión del enfoque biográfico como una perspectiva amplia de investigación, que no se vincula exclusivamente en una tradición disciplinaria, es lo que nos permite presentar los alcances de este enfoque y sus aportes para la enfermería, disciplina donde es posible reconocer algunos trabajos pioneros vinculados a la producción biográfica en salud como la tesis de maestría de Janice Morse realizada a mediados de los años setenta (15).

Como fue mencionado en el párrafo anterior, el enfoque biográfico es una propuesta eminentemente interdisciplinaria, que se desarrolla a partir de las narraciones contenidas en los testimonios recogidos desde los propios sujetos, y por ello su vinculación con los relatos e historias orales. El propio Bertaux aporta en esta línea, que se fortalece luego a partir de las investigaciones de Franco Ferrarotti, Philippe Lejeune y Vincent de Gaulejac $(16,17,18)$.

A finales de los años setenta, la perspectiva biográfica se comienza a usar más frecuentemente en los estudios sobre el poder de la comunidad, desarrollados por Schütze, y en los trabajos de Gabriele Rosenthal y Wolfram Fischer-Rosenthal vinculados a la memoria de las víctimas de violaciones de de- rechos humanos $(19,20,21)$. Ponemos énfasis en este período, ya que las décadas siguientes suponen la consolidación de esta perspectiva como enfoque de investigación, tanto en investigaciones académicas como en la investigación profesional.

En los años noventa se experimenta un auténtico giro hacia lo biográfico, que no implica sólo aspectos metodológicos, sino un cambio de perspectiva teórica, formando parte del movimiento hacia la reflexividad (22).

Tomando en cuenta lo anterior, el enfoque biográfico se constituye en el enfoque teórico metodológico que sustenta la investigación en la que se basa este artículo, el que se construye básicamente a partir de los lineamientos entregados por Daniel Bertaux que buscan hacer visible, a través de sus testimonios, las coordenadas histórico- biográficas de quienes participan en una investigación.

Se trata, por tanto, de un enfoque epistemológico, teórico y metodológico que considera la singularidad y la heterogeneidad de las situaciones individuales, y permite la aparición progresiva de elementos de análisis comunes que estructuran y organizan a determinados colectivos, como ocurre en este caso, con quienes hacen investigación desde el Trabajo Social o la Enfermería.

\section{RESULTADOS}

Usos combinados de materiales biográficos y corrientes en boga.

De las investigaciones de la Escuela de Chicago y de la Escuela Polaca se rescatan para este artículo dos elementos para el análisis de la perspectiva biográfica como enfoque interdisciplinar:

Primero, la diversificación de los materiales de análisis, donde las narrativas no sólo se construyen desde los relatos orales, sino a partir de diversos documentos de vida, que incluye cartas personales, reportes de viaje, diarios personales y fotografías. La penetración actual de las tecnologías de información está permitiendo un uso más activo de los materiales personales en la investigación social, incluyendo aquellos que las personas difunden y comparten en redes sociales (23). Esta perspectiva de usos combinados también se puede rastrear en investigaciones que vinculan experiencias personales de creatividad y apreciación del arte con estudios de la salud (24).

Estos elementos y objetos vinculados a la memoria y los recuerdos animan a los protagonistas de ciertos acontecimientos a escribir y relatar sus experiencias, a rememorar lo acontecido y actualizar sus contenidos. Al mismo tiempo nos recuerda el mandato epistemológico de la investigación biográfica, que contribuye a la memoria y la vinculación entre individuo, estructura e historia.

Lo segundo es la consideración de la experiencia de vida de los sujetos como campo de investigación, posibilidad que resulta 
especialmente pertinente para disciplinas como la Enfermería y el Trabajo Social, que cuentan con el privilegio narrativo de escuchar historias contadas por los sujetos de primera fuente (25).

De esta corriente recogemos la fuerza simbólica del lenguaje y la narración como la dimensión más importante para poder construir y expresar las identidades. Lo anterior es especialmente relevante cuando se constata que en disciplinas como el Trabajo Social o la Enfermería, las narraciones forman parte del quehacer profesional cotidiano (26).

Así se constata en una investigación orientada a desarrollar estos aspectos en estudiantes de enfermería como parte de su aprendizaje clínico. Estudio en el cual los estudiantes hacen saber, a través de sus narraciones, cuáles son los significados que construyen, en el desarrollo de sus prácticas clínicas en los centros sanitarios y cómo éstos les han afectado a las actividades de su vida cotidiana (27).

Acostumbrados a entrevistar y a preguntar a otros, en esta perspectiva de investigación se invierten los papeles habituales y se pide a los otrora entrevistadores que asuman el rol de entrevistados para narrar sus experiencias. De este modo se brinda un espacio que activa la memoria e invita a reflexionar acerca de las vivencias de los propios sujetos y su quehacer, como ocurre también en el trabajo que expone las representaciones sociales acerca de la donación de órganos, publicado en esta revista a fines del año 2016 (28).

Con lo anterior se da cuenta cómo la práctica narrativa y, particularmente, el enfoque biográfico, experimenta una inesperada etapa de éxito y prospectiva en las últimas décadas del siglo pasado. En este período, producto del auge de lo particular y de lo subjetivo, hay un desarrollo y reconocimiento de la escritura experiencial y de los relatos auto-reflexivos, que implican una alteración de las formas de concebir la generación de conocimiento en las ciencias sociales.

Se observa que la subjetividad biográfica llega a aportar conocimiento ya no desde una comprensión a-priori, sino que contiene un giro que implica transitar a un orden de ideas distinto, que entiende el auge contemporáneo de las narrativas, donde la identidad se observa en forma dinámica y flexible, colectiva e individual a la vez.

En este artículo las narraciones son concebidas en el sentido planteado por Guerrero cuando afirma que éstas son plurales en sus perspectivas y principios teóricos; no obstante:

"Los investigadores reconocen ciertos elementos comunes. La narración se caracteriza por poseer un orden y una secuencia de acontecimientos y hechos (...) los narradores crean argumentos de una experiencia desordenada, confiriéndole realidad y coherencia. En relación con esto, los narradores estructuran sus relatos temporal y espacialmente" (29).
La perspectiva biográfico-narrativa nos ayuda a rescatar las visiones que los actores tienen sobre un tema determinado y la forma cómo se pueden ajustar los servicios para responder mejor a las necesidades de los pacientes. En línea se inscriben las investigaciones que recopilan contribuciones de médicos, farmacéuticos, especialistas, matronas, enfermeras, pacientes y usuarios del sistema sanitario. Sus experiencias y vivencias son un elemento clave para la comprensión del funcionamiento de los sistemas de salud y sus posibilidades de mejora.

Al respecto Ortiz señala que los contenidos de estas aproximaciones:

"están directamente relacionados con la ex-
periencia de las personas entrevistadas: las
razones de sus decisiones, las actitudes, sen-
timientos o expectativas ante situaciones
determinadas, los procesos de búsqueda de
asistencia sanitaria y la construcción de sus
identidades profesionales. Algunos de ellos se
acercan a un tema desde la doble perspectiva
de los pacientes y de los profesionales, visión
pluridimensional de notable interés historio-
gráfico" (30).

Tomando en cuenta lo anterior se afirma que los resultados de este trabajo se inscriben en la etapa pluralista de los estudios biográficos y su consolidación como enfoque, al interior del cual es posible reconocer distintas corrientes o tradiciones investigativas, como la corriente americana; francesa, italiana y alemana (31).

En la corriente norteamericana -y también mexicana- es posible reconocer algunas aproximaciones a la noción de trayectorias a partir de los estudios de curso de la vida, dado que esta perspectiva analiza la transición de las personas a lo largo de la vida, centrándose en los movimientos de los individuos y en los modelos temporales que afectan esta transición(32). Esta corriente ha estudiado en profundidad las carreras educativas y sobre todo laborales de los actores sociales; y, por lo mismo, resulta de interés al momento de analizar las transiciones que las personas realizan a lo largo de su vida.

En el ámbito de la salud los estudios de trayectorias suelen seguir los procesos de formación de los profesionales de la salud, así como las trayectorias de pacientes en distintas fases o etapas de su ciclo vital (33).

Por su parte, en Francia tuvieron mayor desarrollo los conceptos de recorrido y trayectoria, los que fueron usados como una línea de investigación específica del enfoque biográfico. La corriente francesa se centra menos en las relaciones de las trayectorias con el ciclo vital y más en las bifurcaciones o puntos de inflexión de las biografías (34). Por tanto, las trayectorias son entendidas como un encadenamiento de acontecimientos que pueden ser pensados en articulación de varias historias o sub-historias: la historia social y política, la historia residencial, la historia familiar, entre otras. 
En esta misma línea, la corriente de estudios francesa ha desarrollado una importante perspectiva en torno a lo inesperado. Estos acontecimientos pueden ser tanto propios de los actores como externos, abordando incluso dimensiones generacionales o colectivas más amplias (35). En el ámbito de la enfermería y los cuidados es posible observar la existencia de trayectorias desviadas o modificadas, producto de enfermedades, accidentes o catástrofes que afectan a sujetos individuales o colectivos. También pueden ser analizadas como "acontecimiento" los accesos a nuevos tratamientos o acceso a prestaciones hasta ese momento no consideradas.

Esta dimensión colectiva tiene mayor relación con los planteamientos de la corriente italiana, cuyo máximo exponente es Franco Ferrarotti, para quien el enfoque biográfico permite el acceso a lo universal y lo general (sociedad) partiendo de los individuos (36). Para este autor es fundamental la comprensión del contexto en que los relatos de vida o las vidas en curso de los sujetos o del grupo tiene lugar, el que se puede abordar, incluso longitudinalmente, dando cuenta de la forma como se articula este problema a lo largo del tiempo.

Los estudios de salud laboral y los accidentes del trabajo pueden ser analizados desde esta corriente, abordando aspectos micro y macrosociales que explican determinados fenómenos o su prevalencia en el tiempo.

Finalmente, en Alemania se expandieron las nociones de historia de vida laboral y proyecto biográfico laboral, en el contexto de los estudios de cultura del trabajo como una forma de hacer referencia a las trayectorias (37). Estas se conciben como secuencias de experiencias que se estructuran en dos dimensiones: la dimensión del tiempo biográfico que establece secuencias típicas según los ciclos de vida y la dimensión del tiempo histórico que ofrece distintas limitaciones y oportunidades que definen espacios sociales diferentes para generaciones distintas.

Concebida de esta forma, la perspectiva biográfica permite la reconstrucción de trayectorias de sujetos individuales y colectivos que son el resultado del entrecruzamiento de tres dimensiones específicas: i) La multiplicidad de elementos presentes en el relato autobiográfico y la importancia que tienen cada uno de ellos; ii) la variabilidad del tiempo en la configuración de la articulación de los elementos presentes en la historia de vida; y iii) la particular articulación de los elementos a lo largo de la misma.

\section{DISCUSIÓN}

Alcances y desafíos para la investigación interdisciplinaria.

Como se ha mostrado en el punto anterior, el enfoque biográfico y el uso de las narrativas en la investigación social pueden ser concebidas como un género de enunciaciones, acciones y representaciones susceptibles de investigar y de constituirse en un campo de investigación interdisciplinario.
Esto es especialmente relevante dado que el enfoque biográfico posee desde su origen una arquitectura interdisciplinar, donde confluyen los aportes de las disciplinas de las ciencias sociales, de los estudios urbanos y las perspectivas socio-históricas (38).

Sus alcances son concebidos en este artículo como un producto o expresión social que se construye principalmente a través de encuentros (entrevistas) biográfico-narrativos, los que se caracterizan por su no estructuración, repetición y genuinidad y que pueden dar origen a historias de vida o relatos biográficos. A diferencia de las historias de vida, los relatos de vida son narraciones biográficas acotadas, por lo general, al objeto de estudio del investigador. Si bien pueden abarcar la amplitud de toda la experiencia de vida de una persona, empezando por su nacimiento, se centran en un aspecto particular de esa experiencia.

Sin entrar en profundidad en el debate sobre la forma cómo se construyen metodológicamente estos relatos biográficos, es preciso reconocer que en este tipo de producciones narrativas lo que se activa es el principio de entrevista reflexiva, donde entrevistado y entrevistador tiene una participación activa como sujetos, en una interacción dialógica (39). En este sentido se asume una perspectiva que presenta similitudes con lo planteado por otros autores, en torno al desarrollo de una práctica reflexiva que interroga y problematiza el propio quehacer en distintos campos profesionales y disciplinarios $(40,41)$.

Lo anterior da cuenta del carácter articulante de los procesos de investigación e intervención y de la idea de práctica reflexiva, que ahora se extiende también del campo investigativo al propio quehacer profesional (42). De este modo se hace énfasis en este artículo a los procesos de reflexión y vigilancia-epistemológica, considerada como un ejercicio de auto-reflexividad disciplinaria que reconoce la importancia de re-pensar y examinar el propio quehacer, sometiéndolo constantemente a revisión, análisis y cuestionamiento (43).

Al mismo tiempo se presta especial atención a la trastienda, a lo que no ha quedado registrado y permanece en un ámbito privado, a las decisiones, a lo no dicho, a los procesos que los investigadores y/o profesionales dejan ocultos cuando desarroIlan su trabajo. Sacarlos a la luz, visibilizar su trastienda, es una las tareas que tiene la investigación desde este enfoque (44).

De esta forma la investigación que se desarrolla y se promueve en este artículo se suma a una corriente reflexiva que han impulsado desde hace tiempo otras disciplinas e investigadores, como las reflexiones que en torno a su quehacer desarrolló en Inglaterra Beatrice Webb, reconocida hoy como precursora del trabajo social, la sociología y la economía (45).

Los desafíos de la investigación cualitativa con enfoque biográfico son múltiples. Particularmente para disciplinas como el Trabajo Social y la Enfermería un primer desafío tiene relación con su reconocimiento o validación como perspectiva de investigación, ya sea en forma combinada con otras técnicas o enfoques o su uso como perspectiva principal en investigaciones que analicen y reflexionen acerca del quehacer disciplinar. 
Un segundo desafío tiene que ver con las posibilidades que brinda este enfoque, tanto para el análisis y comprensión de los fenómenos de investigación de quien investiga, como en el uso reflexivo y terapéutico de quien participa de este proceso. $\mathrm{El}$ carácter terapéutico de la narración constituye una dimensión no muy explorada en este tipo de aproximaciones. El trabajo de White y Epston aporta algunos elementos claves para esta discusión, que requieren ser profundizados, especialmente si se tiene en cuenta el carácter reparador de este tipo de narraciones y sus posibilidades de resignificar eventos traumáticos o dolorosos en la vida de estas personas, como las aproximaciones en torno a este tema ha desarrolladas en la psicología clínica y psicoterapia (46).

Desde el punto de vista de la práctica social, y esencialmente de la relación que se construye entre sujetos, los relatos biográfico son constitutivos y modeladores de la vida de las personas, incluidos los profesionales y los usuarios de los servicios sociales y de salud. "Las narrativas de las personas son realidades discursivas, ancladas histórica y socioculturalmente (...) La narrativa es una forma fundamental que posee el ser humano de dar sentido a una experiencia" (47).

En este sentido, las perspectivas biográficas narrativas también encuentran un tercer desafío cuando se revisan las posibilidades que hoy brindan tecnologías de información para pensar en productos audiovisuales o multimétodos, que han llevado a algunos autores a plantear la noción de "metodología cualitativa audiovisual" (48). En este marco es posible pensar que la narrativa oral se combina con el uso de recursos electrónicos como documentos y correos, imágenes, fotografías, archivos de audio, al mismo tiempo que se recurre a formas de representación de ciertos imaginarios por medio del uso de dibujos, mapas y otras representaciones gráficas.

Finalmente se observa el desafío de inscribir este enfoque de investigación como una práctica recurrente del quehacer profesional, posible de rastrear en el quehacer cotidiano de disciplinas como la Enfermería y el Trabajo Social. En ambas profesiones es habitual encontrar sistemas de registros personales como fichas clínicas y sociales, informes de casos, bitácoras, visitas domiciliarias, datos y memorias institucionales que dan cuenta de los procesos relatados. Todos estos documentos contienen un potencial narrativo-reflexivo que se considera valioso de analizar y de poner en perspectiva de investigación y generación de conocimiento.

\section{CONCLUSIONES}

La realización de esta investigación permitió tener una visión más clara de los usos y posibilidades del enfoque biográfico como perspectiva de investigación en ciencias sociales y de la salud. Los materiales biográficos permiten un uso combinado y muchas veces complementarios con otros enfoques de investigación y ofrecen un amplio campo de análisis para las investigaciones interdisciplinarias.

El enfoque biográfico contiene en su interior distintas corrientes o perspectivas, al interior de las cuales se inscriben de- terminadas líneas de investigación y análisis. Cada corriente contiene un sello que la identifica con un modo de entender la noción de trayectoria y el modo como se estructura la narración que los sujetos hacen sobre ellos mismos

La investigación con enfoque biográfico enfrenta hoy el desafío de adaptar sus procedimientos y formas de análisis a las nuevas tecnologías de información, al mismo tiempo que recupera los elementos tradicionales de este enfoque como la narración oral y los recuerdos de los sujetos, los que hoy pueden ser almacenados en archivos de imágenes, datos y de voz.

Se sugiere estudiar y practicar este enfoque de investigación en los estudios de Enfermería y Cuidados Humanizados, ya que esta perspectiva ofrece nuevas dimensiones de análisis, al mismo tiempo que contribuye a los procesos de reflexividad de los investigadores y/o profesionales acerca de su quehacer.

Cómo citar este artículo: Rubilar Donoso, G. Narrativas y enfoque biográfico. Usos, alcances, y desafíos para la investigación interdisciplinaria Enfermeria (Montev.). [Internet]. 2017 Oct [citado xxxxx]; 6 69-75. Disponible en: https://doi.org/10.22235/ech.v6iEspecial.1453

\section{REFERENCIAS BIBLIOGRÁFICAS}

1. Rubilar G. Prácticas de memoria y construcción de testimonios de investigación. Reflexiones metodológicas sobre autoentrevista, testimonios y narrativas de investigación de trabajadores sociales. Rev. FQS. [Internet] 2015; [citado Mar 2017] 16(3) [112 párrafos]. Disponible en: http:// nbn-resolving.de/urn:nbn:de:0114-fqs150339

2. Thomas W., Znaniecki F. El campesino polaco en Europa y en América. 2da Ed. Madrid: Centro de Investigaciones Sociológicas; 2006.

3. Anderson N. The Hobo: the sociology of the homeless man. Original de 1923.Chicago: Chicago University Press; 2014.

4. Shaw C. The Jack-Roller: A Delinquent Boy's Own Story. Original de 1930. Chicago: Chicago University Press; 1966.

5. Sutherland E. El ladrón profesional. Original de 1937. Madrid: La Piqueta; 1993.

6. Álvarez-Uría F, Varela J. Sociología, Capitalismo y Democracia. 3ra ed. Madrid: Morata; 2004.

7. Addams J. Twenty years at Hull House with autobiographical notes. New York: The MacMillan Company; 1912. [Internet] 2010 [citado Mar 2017] Disponible en: http:// digital.library.upenn.edu/women/addams/hullhouse/huIlhouse.html

8. Álvarez-Uría F, Parra P. The bitter Cry: materiales para una genealogía de la identidad profesional de las pioneras del Trabajo Social en Inglaterra y Estados Unidos. Rev. Cuadernos de Trabajo Social 2014; 27(1): 95-194.

9. Addams J. Hull House maps and paper by the residents of the hull house. New York: T.Y. Crowell; 1895.

10. Nationalities maps [Internet] [citado Mar 2017]. Disponible en: http://hullhousemusseum.org 
11. Zarco J. Estudio introductorio. En: Thomas W, Znaniecki F. El campesino polaco en Europa y en América. 2da ed. Madrid: Centro de Investigaciones Sociológicas; 2006. 22-90.

12. Rojas M. Lo biográfico en sociología. En: Tarrés M. L., coord. Observar, escuchar y comprender. Sobre la tradición cualitativa en la investigación social. México: FLACSO; 2013. 159-207.

13. Denzin N. Interpretative Biography. London: Sage; 1989.

14. Marsal J. Pensar bajo el franquismo. Intelectuales y Política en generación de los años 50. Barcelona: Península; 1979.

15. Bertaux D. y Bertaux-Wiame I. Historias de vida del oficio de panadero. En. Marinas J. y Santamarina C, editores. La historia oral: métodos y experiencias. Madrid: Debate; 1993. 267-281.

16. Morse J. About the author. In: Morse J. Qualitative Health Research: Creating a New Discipline. New York: Routledge; 2016. 173-176.

17. Ferrarotti F. Historie et histoires de vie. La méthode biographique dans les sciences sociales. Paris: Mérididiens Kilncksiecks; 1990.

18. Lejeune Ph. El pacto autobiográfico y otros estudios. Madrid: MeazulEdymion; 1994.

19. de Gaulejac V, Rodríguez S, Tareca E. (coord.) Historia de Vida. Psicoanálisis y Sociología clínica. México: Ediciones UAQ; 2005.

20. Schütze F. Biographieforschung und narratives interviews. Rev. Neue Praxis, 1993; 13( 3): 283-294

21. Rosenthal G. Biographical research. In: Clive S, Gombo G, Gubrium J, Silverman D. editor. Qualitative research practice. London: Sage; 2004. 48-64.

22. Fischer-Rosenthal W. The problem with identity: Biography and solution to some (post) modernist dilemmas. Rev Comenius 1995; (15): 250-265.

23. Bolívar A, Domingo J. La investigación biográfica y narrativa en Iberoamérica: Campos de desarrollo y estado actual Rev. FQS. [internet] 2006; [citado Mar 2017] 7(4). Disponible en: http://www.qualitative-research.net/index.php/ fqs/article/view/161/357

24. Magallanes M, Zanotti A. Análisis etnográfico multiplataforma. Inmersiones en internet y desafíos de campo. Rev FQS [internet]. 2017 [citado Jul 2017] 18(3). Disponible en: http://dx.doi.org/10.17169/fqs-18.3.2645.

25. Jones K. Student nurses in dialogues with hypothetical patients regarding paintings. Rev. Journal of Research in Nursing 2007; vol 12(4): 415-416.

26. Adams TE. A review of narrative ethics. Rev. Qualitative Inquiry 2008 mar; 14(2): 175-194.

27. Rubilar G. Repertorios y aproximaciones biográfico-narrativas. Testimonios y análisis de prácticas investigativas en trabajadores sociales. Rev. FQS [internet]. 2013 [citado Mar 2017] 14(2). Disponible en: http://www.qualitative-research.net/index.php/fqs/article/view/1924/3508

28. Sanjuán A, Cibanal L, Gabaldon E, Cartagena ME, Vizcaya MF, Pérez RM, Domínguez JM, Jurado JL, Lillo M, Domínguez $M$. Enfoque biográfico narrativo como método de investigación para el desarrollo de la inteligencia emocional de los estudiantes de enfermería durante el aprendizaje clínico [Internet]. Alicante: Repositorio Institucional de la Universidad de Alicante [citado Jul 2017]. Disponible en:
https://rua.ua.es/dspace/bitstream/10045/13862/1/3A2Sanjuan08.pdf

29. Ramírez M, Rojas C, Polanco R. Representaciones sociales acerca de la donación de órganos en enfermeros de una unidad de pacientes. Rev. Enfermería: cuidados humanizados 2006; 5(2): 34-40.

30. Guerrero J. Reflexiones sobre la investigación y la práctica narrativa en Trabajo Social. Rev. Alternativa. Cuadernos de Trabajo Social 2014; 21: 63-79. [pagina 69-70]

31. Ortiz T. Fuentes orales e identidades profesionales: las médicas españolas de la segunda mitad del siglo XX. Rev. Asclepio 2005; Vol. LVII: 75-97. [pagina 79]

32. Muñiz Terra L. Los (ex)trabajadores de YPF. Trayectorias laborales a 20 años de la privatización. Buenos Aires: Espacio; 2012.

33. Elder $\mathrm{G}$ Life course dinamics: trayectories and transitions 1968-1980. Ithaca Cornell: University Press; 1985.

34. García A. Las trayectorias del padecimiento: hombres adultos amputados y no-amputados a causa de la diabetes mellitus. Rev. Investigación y Educación en Enfermería 2004; Vol. XXII(2): 28-39.

35. Bidart C. Crises, décisions et temporalites: authors des bifurcations biographiques. Rev. Cahiers internationaux de sociologie 2006; 120: 29-57.

36. Cabanes R, Godard F. (Eds.) Uso de las Historias de Vida en las Ciencias Sociales. Bogotá: Universidad de Externado de Colombia; 1996.

37. Ferrarotti F La Historia y lo cotidiano. Barcelona: Península; 1991.

38. Kholi M. Biografía: relato, texto y método. En Marinas J, Santamarina, C. editores) La historia oral: métodos y experiencias. Madrid: Debate; 1983. 173- 184.

39. Balán J. Las historias de vida en Ciencias Sociales. Teoría y Técnicas. Buenos Aires: Nueva Visión; 1974.

40. Denzin N. The reflexive interview and a performative social science. Rev. Qualitative Research 2001; 1(1): 23-46.

41. Shön D. El profesional reflexivo. Cómo piensan los profesionales cuando actúan. Barcelona: Paidós; 1998.

42. Perrenoud P. Desarrollar la práctica reflexiva en el oficio de enseñar: profesionalización y razón pedagógica. España: Graó; 2004.

43. Shön D. Educating the Reflexive Practitioner. San Francisco: Jossey-Bass; 1987.

44. Bourdieu P, Wacquant L. Transmitir un oficio. Una invitación a la sociología reflexiva. Buenos Aires: Siglo XXI; 2005.

45. Wainerman C, Sautu R. La Trastienda de la investigación social. 3ra ed. Buenos Aires: Ediciones Lumiere; 2004.

46. Webb B. Pasión y Oficio. Rev. REIS 2011; 93(01): 189-201.

47. White M, Epston D. Medios narrativos para fines terapéuticos, Barcelona: Paidós; 1993.

48. Baer A, Schnettler B. Hacia una metodología cualitativa audiovisual El vídeo como instrumento de investigación social. En: Merlino A., ed. Investigación Cualitativa en las Ciencias Sociales: Temas, problemas y aplicaciones. Buenos Aires: Cengage Learning; 2009. 149-173.

49. Guerrero J. Reflexiones sobre la investigación y la práctica narrativa en Trabajo Social. Rev. Alternativa. Cuadernos de Trabajo Social 2014; 21: 63-79. [página 65] 